\title{
Structure and Function of the Proteasome Activator PA28 of the Malaria Parasite Plasmodium falciparum
}

Riley Metcalf ${ }^{1}$, Eric Hanssen ${ }^{1,2}$, Stanley C. Xie ${ }^{1}$, David Gillett ${ }^{1}$, Andrew Leis ${ }^{2}$, Craig Morton ${ }^{1}$, Michael W Parker ${ }^{1,3}$, Wilson Wong ${ }^{4}$, Michael Griffin ${ }^{1}$ and Leann Tilley ${ }^{1^{*}}$

${ }^{1 .}$ Department of Biochemistry and Molecular Biology, The University of Melbourne, Melbourne, Australia.

2. Advanced Microscopy Facility, Bio21 Molecular Science and Biotechnology Institute, The University of Melbourne, Melbourne, Australia.

3. Australian Cancer Research Foundation Rational Drug Discovery Centre, St Vincent's Institute of Medical Research, Fitzroy, Victoria, Australia.

4. The Walter and Eliza Hall Institute of Medical Research, 1G Royal Parade, Parkville, Australia.

* Corresponding author: 1tilley@unimelb.edu.au

The proteasome is a multi-subunit enzyme complex that is responsible for most of the non-lysosomal proteolysis in eukaryotic cells, underpinning proteostasis and regulating key processes such as the cell cycle. The $20 \mathrm{~S}$ proteasome core is comprised of two heptameric rings of $\beta$-subunits, containing the catalytic sites, sandwiched by two heptameric $\beta$-rings. Substrate access to the proteasome catalytic sites is restricted by $\mathrm{N}$-terminal sequences of the $\alpha$-subunits (1). The activity of the $20 \mathrm{~S}$ proteasome is regulated by protein complexes that bind to one or both ends of the proteasome. The most wellcharacterised activator is the 19S complex [1]. Another activator the 11S or PA28 complex is involved in ubiquitin-independent degregation. The malaria parasite, Plasmodium falciparum, is heavily reliant on its proteasome and proteostasis machinery.

Here we recombinantly expressed and purified an orthologue of the PA28 regulator (PfPA28) from Plasmodium falciparum. The structure of PfPA28 was solved at $3.1 \AA$ by X-ray crystallography, revealing a heptameric bell-shaped structure. We also purified Pf20S proteasome from parasite cultures and showed by Native-PAGE that PfPA28 readily forms single- and double-capped complexes with $P f 20$ S. Moreover, we demonstrated the stimulatory effects of PfPA28 on Pf20S activity using fluorogenic peptide substrates. We next structurally characterized the PfPA28-Pf20S complex using cryo-EM. Samples $(500 \mu \mathrm{g} / \mathrm{mL})$ were prepared on quantifoil grids and vitrified before collection of data using a Talos Arctica with a K2 camera, and analysis of 126,000 particles. We solved the structure of both the single capped and the double capped Pf20S to respectively, 3.9 and $3.8 \AA$ (Figure 1). The activation loop (between helices 2 and 3 in each PfPA28 monomer) plays a primary role in the interaction with the $\alpha$-rings of Pf20S. In addition, the flexible C-terminal tails of PfPA28 insert into the hydrophobic pockets between $\alpha$-subunits and become ordered upon complex formation. The interaction induces conformational changes in $\alpha$-subunits consistent with opening of the $\alpha$-annulus, to allow substrate entry to the proteasome catalytic chamber. In the absence of PfPA28, the pore is blocked by the $\alpha 2-4$ subunit N-termini. The $\alpha 5-7$ and $\alpha 1 \mathrm{~N}$-termini adopt an 'open' state both with and without PfPA28. Long disordered loops ( $>50$ residues between helices 1 and 2 in each monomer) at the distal side of the PfPA28 heptamer are evident in Small Angle X-ray Scattering (SAXS) data and observed in cryoEM class averages, with parts of this one loop resolved by X-ray crystallography in some subunits of the crystal structure. These loops may form an entropic brush that controls the entry of substrates to the highly basic entry pore. We used multi-body refinement [2] to study the dynamics of the PfPA28/core particle complex. The resulting Eigenvectors describe a Gaussian motion of the PfPA28 
along the top of the $\alpha$ subunit ring indicative of a random non-correlated motion. Molecular dynamics simulation support conformational flexibility of the PfPA28 heptamer

In summary, PfPA28 binds to the $\alpha$ subunits, destabilizing the closed conformation of the pore and opening the pore (Figure 2). We propose that lateral transfer of substrates through this interface is an alternative route of substrate egress, particularly in hydride 19S/20S/11S complexes.

References:

[1] D Voges, P Zwickl and W Baumeister, Annu Rev Biochem 68 (1999), p.1015

[2] J Zivanov et al., eLife (2018) p. e42166.

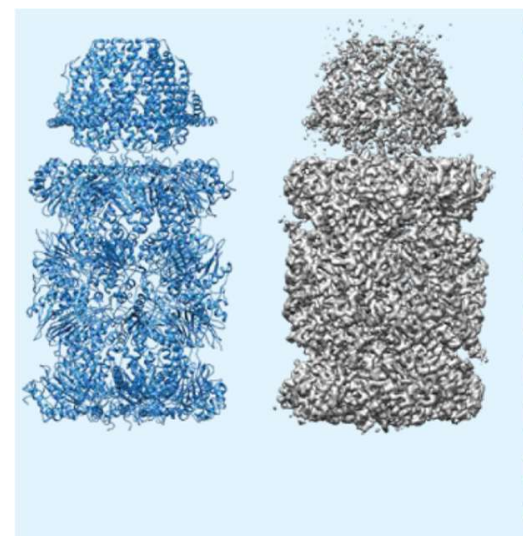

Single-PfPA28 cap $3.9 \AA$
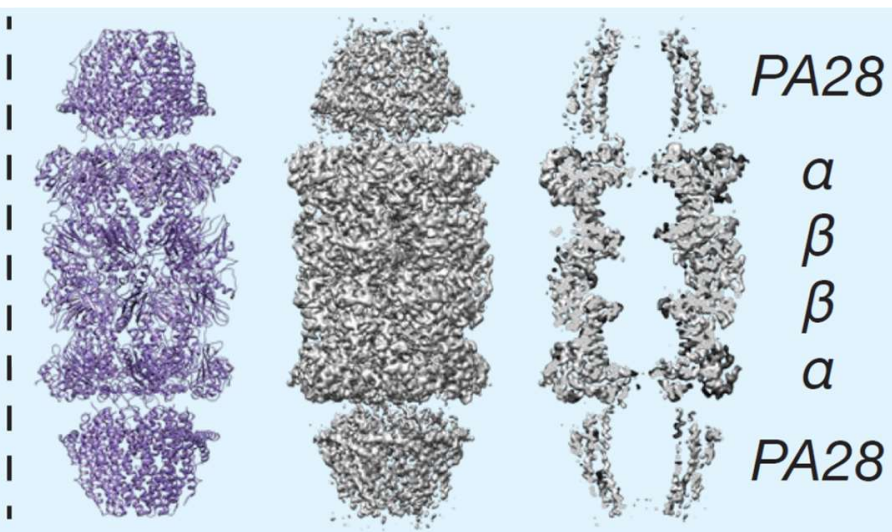

Double-PfPA28 cap

$3.8 \AA$

Figure 1. Structures of the single and double capped PfPA28/Pf20S complexes and corresponding molecular models.

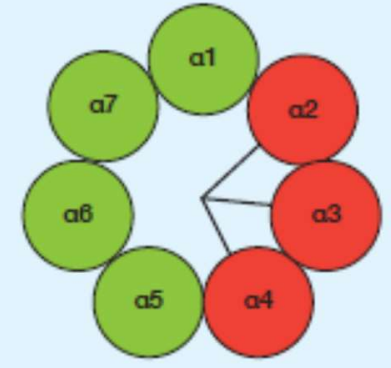

PfPA28 unbound.

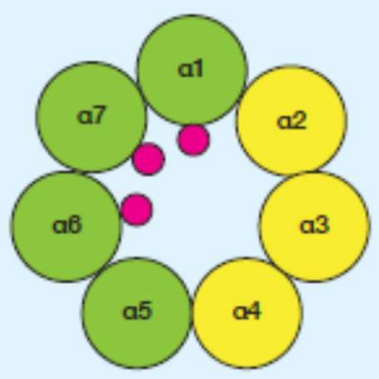

PfPA28 bound.

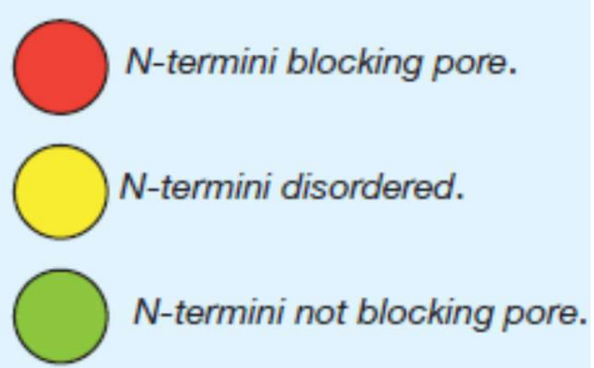

PA28 activation loop. [Strong PA28 binding.]

Figure 2. Schematic of the closed and open conformation of the pore of the Pf20S proteasome. 